\title{
Die Schönheit der molekularen Architektur von Viren und ihre Entschlüsselung durch Beugungsmuster
}

\author{
Gero Friesecke
}

Meine Nicht-Mathematiker-Freunde runzeln oft die Stirn, wenn ich das Wort Schönheit in Zusammenhang mit Viren verwende. Schließlich befallen diese kleinen Monster tagtäglich Menschen, Tiere und Pflanzen, und begehen schreckliche Untaten bis hin zur Tötung ihrer Wirte. Gleichwohl sind viele Viren Miniatur-Bauwerke der $\mathrm{Na}$ tur von faszinierender geometrischer Gestalt, elegant zusammengesetzt aus nur wenigen Bausteinen oder gar nur einem einzigen.

Ein bemerkenswerter Unterschied zu den LegoBauwerken von Kindern ist die Umkehr des Prinzips einfacher Baustein, kompliziertes Gebäude. Die Bausteine sind komplizierte, unregelmäßige, große Biomoleküle (Proteine), die dem naiven Betrachter als wirrer Haufen von 500 bis 5000 Atomen erscheinen. Nie würde man erwarten, was in der Natur tatsächlich passiert: Die Bausteine setzen sich durch Selbst-Assemblage zu einem einfachen, regelmäßigen geometrischen Gebilde zusammen.

\section{Helices und Ikosaeder}

Im Falle des Tabakmosaikvirus (TMV) ergibt sich eine Helix. Mathematisch ist eine solche Protein-Helix wie folgt definiert: Die Atompositionen sind der Orbit der Atompositionen im Protein unter einer wiederholt angewendeten Schrauben-Transformation, d.h. einer Drehung des Proteins um einen bestimmten Winkel, gefolgt von einer Verschiebung entlang der Drehachse. Beim TMV ist die Schraubentransformation eine Drehung um $49 / 3 * 360^{\circ}$ um die senkrechte Achse, gefolgt von einer Verschiebung nach oben um $1,4 \AA$.

Eine etwas kompliziertere Architektur findet sich z. B. beim Herpes Simplex. Die Proteine bilden ein Ikosaeder mit leicht nach außen ausgebeulten Seitenflächen. Jede Seitenfläche besteht - inklusive der Kanten - aus $5+4+3+2+1=15$ Proteinen.

Fassen wir die Proteine als Knoten einer Triangulierung auf, so haben wir es mit einer interessanten Triangulierung der Sphäre zu tun. Aus der numerischen Mathematik oder der diskreten Geometrie wissen wir, dass eine solche Triangulierung ab einer gewissen Feinheit notwendigerweise „Defekte“ enthalten muss. Dies ist der Tatsache geschuldet, dass die Sphäre - im Gegensatz zum durch Helix-Strukturen triangulierten Zylinder - nichtverschwindende intrinsische (Gauß-)Krümmung besitzt. Die Defekte sind in unserem Fall gerade die Ecken der Seitenflächen. So bildet etwa das vierte Protein von oben die obere Ecke der vorderen Seitenfläche. Dieses Protein hat nur fünf statt sechs Nachbarn. Genaueres Betrachten zeigt, dass es sich übringens auch um ein anderes Protein handelt: Es besitzt näherungsweise eine fünffache anstatt einer sechsfachen Symmetrie um die Achse senkrecht zur Virusoberfläche.

Die in Bild I und 2 dargestellten Viren zeigen eine hohe mathematische Regelmäßigkeit. Interessanterweise sind solche Architekturen aber nicht die Ausnahme, sondern die Regel. Nahezu alle kleinen Viren, deren molekulare Struktur bisher aufgeklärt werden konnte, sind entweder
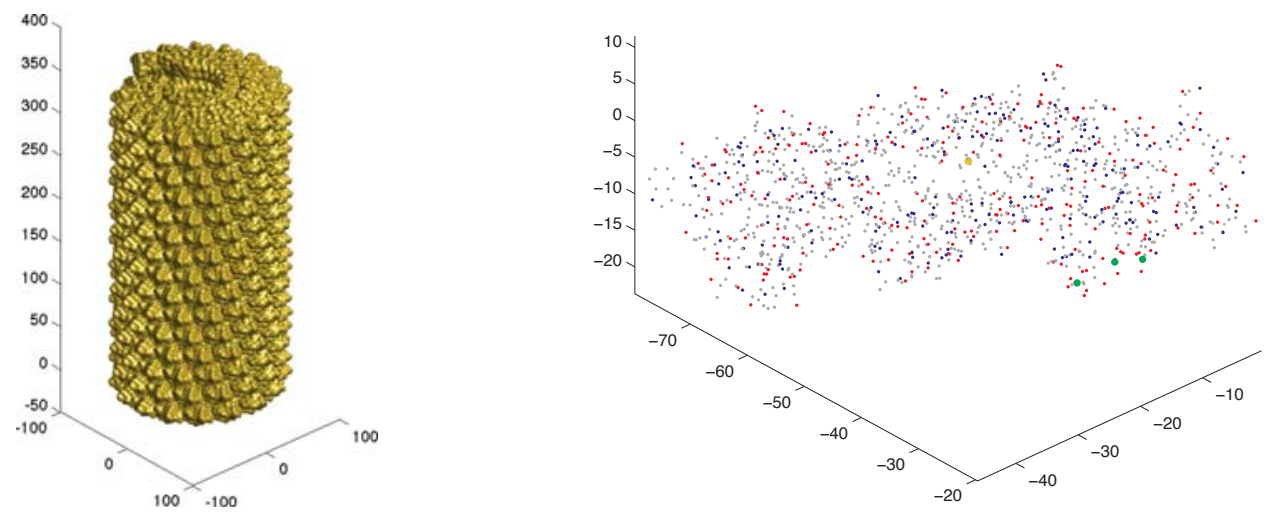

Abbildung I. Ein Tabakmosaikvirus (links) und sein Baustein (rechts). Der Baustein ist ein Protein aus 1284 Atomen (Wasserstoff nicht mitgezählt): $794 \times$ Kohlenstoff (grau), $260 \times$ Sauerstoff (rot), $226 \times$ Stickstoff (blau), I $\times$ Schwefel (orange), $3 \times$ Phosphor (grün). Das Virus ist eine aus identischen Kopien dieses Proteins zusammengesetzte Helix. Das Bild zeigt 245 Proteine, das gesamte Virus hat etwa die fünffache Länge. Koordinatenachsen in Ångström $\left(10^{-10} \mathrm{~m}\right)$. (Daten aus Ref. [I], Visualisierung des Autors) 


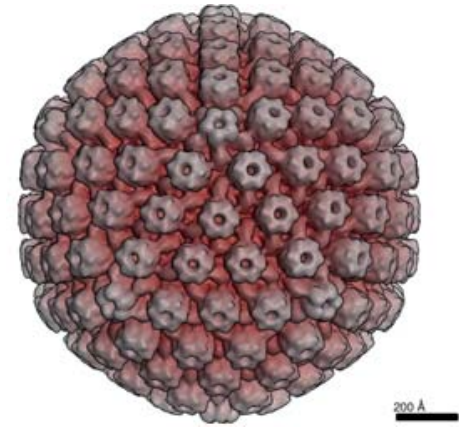

Abbildung 2. Herpes Simplex-Virus Typ IB (Daten aus Ref. [2], Visualisierung: Jean-Yves Sgro, University of Wisconsin-Madison [3], reproduziert mit Genehmigung)

Helices, oder sie haben eine sphärische Gestalt mit der Symmetrie eines regelmässigen Polyeders. Dies wirft eine ganze Reihe fundamentaler Fragen auf - nicht nur an die Biologie und die Physik, sondern auch an die Mathematik.

Wieso ordnen sich die Proteine symmetrisch an? Oder, tiefergehend gefragt: Wieso haben sich eigentlich Viren entwickelt, deren Proteine sich derart anordnen - welchen biologischen Vorteil hat das? Woher und wie sicher wissen wir überhaupt, dass Viren und ihre Proteine tatsächlich die in den Schaubildern gezeigte atomare Struktur haben? Kann man die nicht-kristallinen Symmetrien von Viren ausnutzen, um - analog zur Röntgenstrukturanalyse von Kristallen - bildgebende Verfahren mit atomarer Auflösung zu entwickeln? Mehr zur letzten Frage im Abschnitt Ein neuer Twist.

Die erste Frage ist eine rein mathematische, wenn man die physikalischen Wechselwirkungskräfte zwischen den Proteinmolekülen als gegeben nimmt. Genauer gesagt haben wir es mit einer Variante des Kristallisationsproblems zu tun: Wieso führen interatomare Wechselwirkungen bei hinreichend niedriger Temperatur fast immer zu kristalliner (bzw. helikaler, ikosaedrischer, ...) Ordnung? Dies ist bisher selbst für einfache Modellwechselwirkungen mathematisch nicht bewiesen. Das Kristallisationsproblem ist eng mit Packungsproblemen verwandt. Auch der beeindruckende computergestützte Beweis der Kepler-Vermutung durch Thomas Hales hat nichts an der Tatsache geändert, dass wir die Vorzüge periodischer Ordnung beim Kugelpacken in drei Dimensionen bisher nicht wirklich verstehen.

Die Frage nach dem biologischen Nutzen symmetrischer Proteinanordnungen haben bereits James Watson und Francis Crick in den 1950er Jahren beantwortet, und zwar bevor solche Symmetrien experimentell nachgewiesen waren. Sie sagten solche Anordnungen mit dem folgenden Argument voraus: Die Virushülle muss groß genug sein, um die RNA des Virus einzuschließen; hierfür ist es günstiger, viele identische Kopien eines kleinen Proteins als ein großes zu verwenden, da ansonsten zur Kodierung mehr RNA benötigt würde, als in die Hülle hereinpasst. Dass aus dem Prinzip viele identische Proteine die
Konsequenz regelmäßige Anordnung folgt - also die erwartete Lösung unseres Kristallisationsproblems - setzten Watson und Crick als plausibel voraus: Whenever [...] a structure of a definitive size and shape has to be built from smaller units $[. .$.$] the packing arrangements are likely to be$ repeated again and again - and hence [...] the sub-units are likely to be related by symmetry elements.

Zu unserer nächsten Frage - Woher und wie sicher kennen wir die atomare Struktur gewisser Viren und Proteine? - lässt sich weit mehr sagen. Wie funktionieren bildgebende Verfahren mit atomarer Auflösung, und welche Mathematik steckt dahinter?

\section{Röntgenkristallografie}

Die zentrale Methode zur Aufklärung von Proteinstrukturen ist die Röntgenkristallografie (x-ray crystallography). Neunzig Prozent der heute bekannten Strukturen wurden auf diese Weise bestimmt. Man isoliert das Protein, kristallisiert es - d. h. man stellt aus identischen Kopien des Proteins künstlich eine Kristallgitterstruktur [4] her -, sendet Röntgenstrahlen mit Wellenlänge der GröBenordnung der Atomabstände (also $10^{-10} \mathrm{~m}$ ) auf den Kristall, misst das resultierende Beugungsmuster, d. h. die Intensität der emittierten Strahlung, versucht mithilfe geeigneter Algorithmen oder Modellierungsannahmen die - aus dem Beugungsmuster nicht ersichtlichen - Phasen der emittierten Strahlung zu rekonstruieren (phase retrieval: Eine mathematische Formulierung dieser Aufgabenstellung findet sich im letzten Abschnitt) und kann aus diesen Daten schließlich die atomare Struktur ablesen.

Entscheidend für die atomare Auflösung dieser Methode, d.h. die Sichtbarkeit der einzelnen Atompositionen, ist die Tatsache, dass die von einer Kristallstruktur emittierte Strahlung aus diskreten Peaks besteht, deren Intensität um den Faktor $10^{2}$ bis $10^{4}$ oberhalb des Rauschpegels liegt. Mathematisch kann dieser bemerkenswerte Effekt als Manifestation der Poisson'schen Summenformel der Fourier-Analysis verstanden werden (s. letzter Abschnitt). Die Entdeckung der Röntgenbeugung am Kristall im Jahre 1912 brachte Max von Laue 1914 den schnellsten Nobelpreis aller Zeiten ein; seither folgten I 3 Nobelpreise, u. a. für die erste erfolgreiche Bestimmung einer Kristallstruktur (Vater und Sohn: W. Henry Bragg und W. Lawrence Bragg 1915) bzw. einer Proteinstruktur (Max Perutz und John Kendrew 1962). Das erste entschlüsselte Protein war das Myoglobin, das die Speicherung von Sauerstoff in unseren Muskeln ermöglicht. Heutzutage ist die Struktur von etwa 100000 Proteinen bekannt; diese bilden jedoch nur einen Bruchteil aller Proteine. Ein zentrales Problem der Röntgenkristallografie liegt in der Schwierigkeit, ein Protein, dessen biologische Funktion darin besteht, robust eine nicht-kristalline Struktur, etwa eine Helix oder ein Ikosaeder zu bilden, in ein Kristallgitter zu zwingen. Dies ist in der Praxis oft nicht möglich, oder verändert wichtige Details der atomaren Struktur. 
Faserbeugung

Eine weniger invasive Methode, die zumindest bei Helixstrukturen wichtige Erkenntnisse geliefert hat, aber eine geringere Auflösung besitzt, ist die Variante der Faserbeugung (fiber diffraction). Man analysiert direkt die Röntgenbilder der nativen Helixstruktur.

Hier liegt die Schwierigkeit in der Interpretation der Daten: Das Röntgenmuster ist nur noch in der Richtung der Helixachse diskret - auch eine Helixstruktur wie TMV besitzt noch diskrete Translationssymmetrie in Achsenrichtung -, senkrecht dazu aber ist das Muster kontinuierlich. Das Signal ist auf sogenannten layer lines konzen-

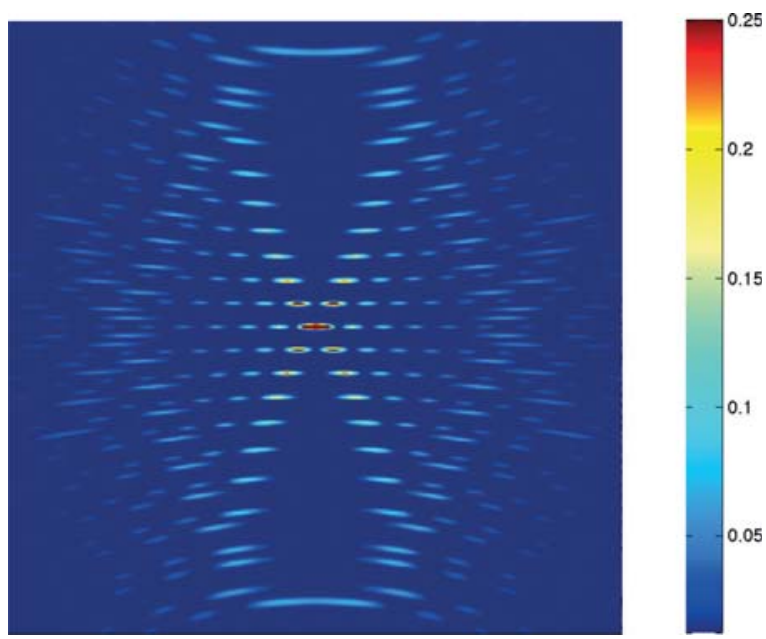

Abbildung 3. Röntgenmuster der TMV-Helix (Simulation des Autors). Zur besseren Veranschaulichung wurde die Elektronendichte jedes Proteins hier durch eine Delta-Funktion modelliert; das volle Muster kommt durch Interferenz von 1284 in Horizontalrichtung gegeneinander dilatierter solcher Muster zustande. Als einfallende Strahlung wurde eine ebene Welle gewählt (Wellenlänge I.54 A). Das Bild zeigt die Intensität der emittierten Strahlung gemäß Gleichung (3) der Formelsammlung auf S. 212.

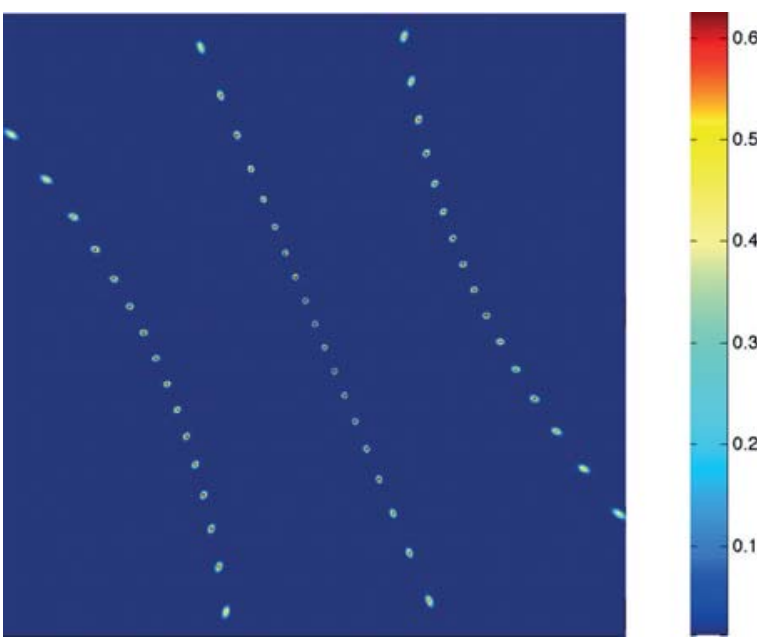

Abbildung 4. Röntgenmuster der ebenen Schicht, die sich durch Aufschlitzen und Flachbiegen der TMV-Helix ergibt (Simulation des Autors). triert. Ein Computerexperiment zeigt, dass der Verlust der Diskretheit des Signals, und damit der vollen atomaren Auflösung, allein durch die Krümmung der Proteinanordnung verursacht wird, und nicht etwa durch ihre Zweidimensionalität. Schlitzen wir den TMV-Zylinder längsseits auf und biegen ihn zu einer ebenen Schicht auseinander, so ergibt sich ein einfaches diskretes Punktmuster (Bild 4).

Im Falle des TMV konnte die Struktur des Proteins mithilfe der Faserbeugungsmethode trotzdem ziemlich genau bestimmt werden. Die Liste der Forscher, die hierzu beigetragen haben, liest sich wie ein Who's Who der Gründerzeit der Strukturbiologie: John Bernal, Rosalind Franklin, Don Caspar, Aaron Klug. Das hier gezeigte erste volle atomare Modell wurde von Gerald Stubbs und Mitarbeitern entwickelt [I]; die Genauigkeit ist dort mit 2.9 Ångström angegeben.

\section{Ein neuer Twist}

Startpunkt meiner eigenen Beschäftigung mit dem Thema Strukturaufklärung durch Röntgenmuster war die folgende Frage, die ich mir zusammen mit Richard James (University of Minnesota) und Dominik Jüstel (TUM) gestellt habe: Kann man die Vorteile der Röntgenkristallografie und der Faserbeugung kombinieren? Ist es also möglich, durch Abänderung der einfallenden Strahlung aus einer nativen nicht-kristallinen Struktur diskrete Röntgenmuster, und damit ein bildgebendes Verfahren mit atomarer Auflösung, zu erhalten?

Prinzipiell konnten wir diese Frage kürzlich im Falle von Helixstrukturen positiv beantworten [5, 6]: Indem man die einfallende Strahlung geeignet abändert, erhält man diskrete Muster. Wir bestimmen die entsprechende Strahlung mathematisch als exakte Lösung der MaxwellGleichungen und nennen sie aufgrund ihrer geometrischen Struktur twisted waves.

Wie im Kristallfall kann die atomare Struktur aus dem Beugungsmuster bestimmt werden - durch Ausnutzung der Peak-Struktur sowie Verwendung geeigneter phaseretrieval-Algorithmen. Wir demonstrieren dies, indem wir die Struktur des PfI-Virus (Protein Data Bank, Datensatz I Pfi) aus seinem simulierten Beugungsmuster unter twisted waves rekonstruieren [6].

Dies eröffnet die Perspektive, dass die Strukturbestimmung zumindest bei helixförmigen Viren und Proteinen eines Tages in vivo stattfinden könnte: ohne künstliche Kristallisation, aber trotzdem mit atomarer Auflösung.

Aber auch hier gibt es einen Haken: Die Strahlung ist in der Praxis im benötigten kurzwelligen Bereich derzeit nicht herstellbar. Ähnliche Strahlen mit optischer Wellenlänge sind allerdings unter dem Begriff orbital angular momentum light seit den Neunzigerjahren bekannt [8], und die produzierbaren Wellenlängen sind heutzutage 


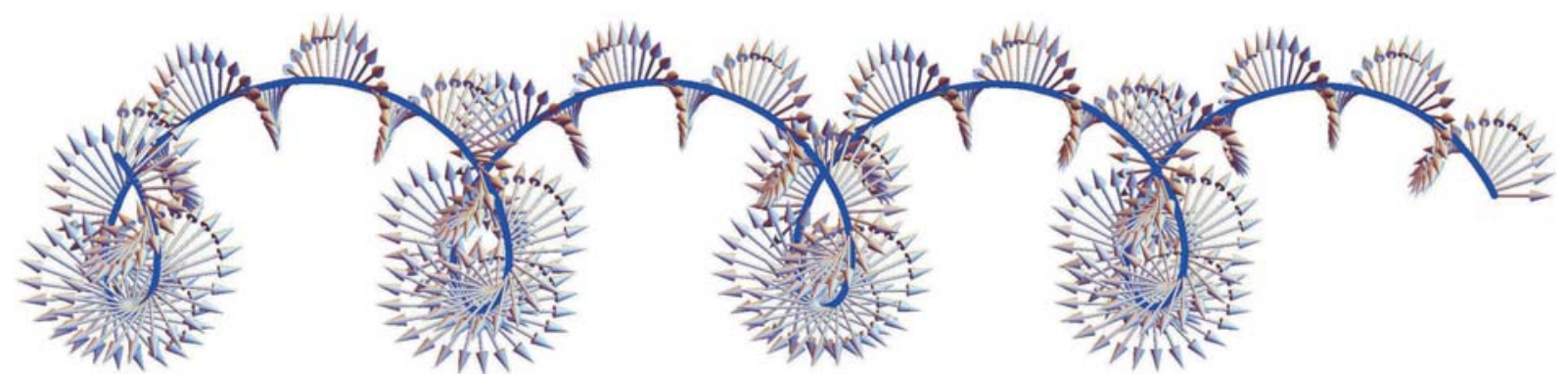

Abbildung 5. Eine twisted wave: mathematisch eine exakte Lösung der Maxwell-Gleichungen, die diskrete Röntgenmuster von Helixstrukturen erzeugt. Siehe Gl. (6) der Formelsammlung. Das Bild (Original: Ref. [6], reproduziert mit Erlaubnis der International Union of Crystallography, http:/ljournals.iucr.org) zeigt den elektrischen Feldvektor entlang einer Kurve, die tangential zur Richtung des Energietransports, des sogenannten Poynting-Vektors, verläuft.

nur noch um einen Faktor 100 vom benötigten Bereich entfernt.

\section{Mathematische Prinzipien}

Zum Abschluss sollen die mathematischen Prinzipien erläutert werden, die den Röntgenmustern (Bild 3 und 4) und der twisted wave (Bild 5) zugrundeliegen. Röntgenmuster entstehen durch das Zusammenspiel von vier grundlegenden physikalischen Prozessen, die alle berücksichtigt werden müssen, um ein vorhersagekräftiges mathematisches Modell zu erhalten: Röntgenstrahlen werden eingeschaltet und treffen auf eine Materialprobe; die einfallende Strahlung induziert eine oszillatorische Bewegung der Elektronenwolke des Materials; diese Bewegung produziert ihrerseits elektromagnetische Strahlung; die Intensität der emittierten Strahlung wird von Detektorzellen registriert. Letztere sind typischerweise auf einem Bildschirm hinter der Probe angebracht; daher die zweidimensionalen Intensitätsmuster in Experimenten oder in den Computersimulationen in Bild 3 und 4.

Eine mathematische Beschreibung ergibt sich wie folgt. Für die Entstehung von Streumustern ist die Wellennatur der einfallenden Röntgenstrahlen verantwortlich. Die Strahlen müssen folglich als elektromagnetische Wellen aufgefasst werden. Darüber hinaus müssen sie über räumliche und zeitliche Abstände hinweg eine feste Phasenbeziehung haben (Kohärenz). Erzeugt werden kohärente Wellen beispielsweise von Lasern oder einem Synchroton. Mathematisch entsprechen solche Wellen zeitharmonischen Lösungen der Maxwell-Gleichungen im Vakuum (siehe (I) und (2)). Die beiden Vektorfelder $E$ und $B$ beschreiben das elektrische Feld und das damit gekoppelte Magnetfeld; die Zeitharmonizitätsbedingung (2) beschreibt die Kohärenz. Die Konstante $c$ ist die Lichtgeschwindigkeit. Quantenmechanische Effekte können bei den in der Röntgenkristallografie verwendeten Feldstärken und Wellenlängen vernachlässigt werden.
Die Modellierung der weiteren physikalischen Prozesse führt auf das grundlegende Modell (3) für die Intensität der emittierten elektromagnetischen Strahlung. Der wichtigste Bestandteil ist das Integral eines Dreifachproduktes aus dem einfallenden elektrischen Feld, der Elektronendichte der Materialprobe sowie der in Richtung des Messpunktes $x$ emittierten Welle. Die Formel beruht auf asymptotischen Näherungen (z. B. Fernfeld) und liefert trotzdem eine bemerkenswert akkurate Übereinstimmung mit experimentellen Röntgenmustern.

An dieser Stelle sei angemerkt, dass das Gesetz (3) eine interessante Brücke zwischen fundamentaler Physik, Fourier-Analysis und mathematischer Datenanalyse schlägt. Zunächst zur Fourier-Analysis: Ist die einfallende Strahlung eine ebene Welle (5), so reduziert sich das Integral in (3) auf eine Fourier-Transformation der Elektronendichte. Anders gesagt: In der Physik von Röntgenmustern ist die mathematische Definition der FourierTransformation versteckt!

Nun zur Datenanalyse. Vergessen wir für einen Augenblick die Physik und nehmen einen rein datenwissenschaftlichen Standpunkt ein. Welche Daten über eine Materialprobe liefert eigentlich die Röntgenkristallografie? Im Fall eben einfallender Röntgenwellen und der Idealisierung, dass die Intensitätsmuster $(I(x)$ aus Gl. (3)) für alle Wellenlängen - d.h. alle Wellenvektoren in (5) - vorliegen, ist der Datensatz gerade der Absolutbetrag der Fourier-Transformierten der Elektronendichte. Die Phase der Fourier-Transformierten geht aber verloren. Die Extraktion der atomaren Struktur der Materialprobe, d.h. der Elektronendichte, aus den Daten reduziert sich somit auf das in der Bild- und Datenanalyse berühmtberüchtigte mathematische Phasenproblem: Rekonstruiere eine Funktion aus dem Absolutbetrag ihrer FourierTransformierten!

Obwohl das Gesetz (3) nur Standardwissen wiederspiegelt, hat uns die Herleitung viel Zeit gekostet (siehe [7] sowie eine in [5] gegebene Zusammenfassung): Leider sind nämlich nur Teile und Spezialfälle sowie zahlreiche inkorrekte Versionen in der Literatur zu finden.' Typischer- 


\section{Formelsammlung Röntgenkristallographie}

Maxwell'sche Gleichungen

$$
\frac{1}{c^{2}} \frac{\partial \mathbf{E}}{\partial t}=\operatorname{curl} \mathbf{B}, \operatorname{div} \mathbf{E}=0, \frac{\partial \mathbf{B}}{\partial t}=-\operatorname{curl} \mathbf{E}, \operatorname{div} \mathbf{B}=0
$$

Zeitharmonische Lösungen

$$
\text { Lösungen der Form } \mathbf{E}(\mathbf{x}, t)=\mathbf{E}_{0}(\mathbf{x}) e^{-i \omega t}, \mathbf{B}(\mathbf{x}, t)=\mathbf{B}_{0}(\mathbf{x}) e^{-i \omega t}
$$

Intensität der emittierten Strahlung ( $\rho$ Elektronendichte der Materialprobe)

$$
I(\mathbf{x})=\frac{1}{|\mathbf{x}|^{2}}\left|\left(\mathbf{I}-\frac{\mathbf{x} \mathbf{x}^{T}}{|\mathbf{x}|^{2}}\right) \int_{\mathbb{R}^{3}} \mathbf{E}_{0}(\mathbf{y}) \rho(\mathbf{y}) e^{-i \mathbf{k}^{\prime}(\mathbf{x}) \cdot \mathbf{y}}\right|^{2}, \mathbf{k}^{\prime}(\mathbf{x})=\frac{\omega}{c} \frac{\mathbf{x}}{|\mathbf{k}|}
$$

Operation euklidischer Transformationen $g(x)=R x+b, R$ Rotation, $b$ Translationsvektor, auf skalaren Funktionen und Vektorfeldern

$$
(g \rho)(\mathbf{x})=\rho\left(R^{-1}(\mathbf{x}-\mathbf{b})\right),\left(g \mathbf{E}_{0}\right)(\mathbf{x})=R \mathbf{E}_{0}\left(R^{-1}(x-b)\right)
$$

Ebene Wellen (plane waves) ( $\mathbf{k}_{0}$ Wellenvektor, $\mathbf{n}$ Polarisationsrichtung); diese lösen (I), wenn $\mathbf{k}_{0} \cdot \mathbf{n}=0,\left|\mathbf{k}_{0}\right|=\frac{\omega}{c}$

$$
\mathbf{E}(\mathbf{x}, t)=\mathbf{n} e^{i\left(\mathbf{k}_{0} \cdot \mathbf{x}-\omega t\right)}, \mathbf{B}(\mathbf{x}, t)=\frac{1}{\omega}\left(\mathbf{k}_{0} \times \mathbf{n}\right) e^{i\left(\mathbf{k}_{0} \cdot \mathbf{x}-\omega t\right)}
$$

Twisted waves (Zylinderkoordinaten $x=(r \cos \varphi, r \sin \varphi, z) ; J_{\alpha}$ Besselfunktion; $\alpha$ ganzzahlig); diese lösen (I), wenn $(0, \gamma, \beta) \cdot \mathbf{n}=0,|(0, \gamma, \beta)|=\frac{\omega}{c}$

$$
\mathbf{E}(r, \varphi, z, t)=e^{i(\alpha \varphi+\beta z-\omega t)}\left(\begin{array}{ccc}
\cos \varphi & -\sin \varphi & 0 \\
\sin \varphi & \cos \varphi & 0 \\
0 & 0 & 1
\end{array}\right)\left(\begin{array}{ccc}
\frac{n_{1}+i n_{2}}{2} & \frac{n_{1}-i n_{2}}{2} & 0 \\
\frac{n_{2}-i n_{1}}{2} & \frac{n_{2}+i n_{1}}{2} & 0 \\
0 & 0 & n_{3}
\end{array}\right)\left(\begin{array}{c}
J_{\alpha+1}(\gamma r) \\
J_{\alpha-1}(\gamma r) \\
J_{\alpha}(\gamma r)
\end{array}\right)
$$

weise wird in der Literatur mit der Vereinfachung gearbeitet, einfallende und emittierte Strahlung mit der skalaren Wellengleichung statt den Maxwell-Gleichungen zu modellieren sowie die einfallende Strahlung von vornherein als ebene Welle anzunehmen. Die Dipol-Natur der emittierten Strahlung - beschrieben durch den matrixwertigen Vorfaktor des Integrals - geht auf diese Weise verloren, und Röntgenwellen, in denen nicht nur die Länge, sondern auch die Richtung des elektromagnetischen Feldvektors fluktuiert, wie die twisted waves in Bild 5, können nicht erfasst werden.

Dass die Formel (3) auf diskrete Peakmuster wie in Bild 4 führt, wenn ebene Wellen (5) auf Kristallstrukturen treffen, ergibt sich mit besonderer Klarheit im idealisierten Fall einer unendlich ausgedehnten Kristallstruktur, d.h. einer in drei (oder, im Falle ebener Schichten wie dem Material Graphen, zwei) linear unabhängige Richtungen periodischen Elektronendichte. Die Poissonsche Summenformel der Fourier-Analysis (siehe etwa [9]) besagt, dass nun das Integral für alle Werte des einfallenden Wellenvektors außerhalb einer diskreten Menge exakt verschwindet. Diese in höchstem Maße bemerkens- werte mathematische Tatsache ist in den - an diskreten Peaks konzentrierten - Röntgenmustern von Kristallen physikalisch beobachtbar.

So weit, so gut. Aber wie kommt man auf twisted waves (6), mit ihrer merkwürdigen Rotation des Feldvektors, verursacht durch die Rotationsmatrix in der Formel?

Wir gehen zunächst einer einfacheren Frage nach: Wie kommt man eigentlich auf ebene Wellen? Angenommen, sie wären uns nicht a priori, als die von StandardRöntgenquellen emittierte Strahlung, gegeben. Könnten wir sie aus dem Ziel herleiten, dass die Röntgenmuster von Kristallstrukturen diskret sein sollen?

Für eine solche Herleitung ist das bisherige Verständnis der Diskretheit der Muster (z. B. mithilfe der Poissonschen Summenformel) nicht ausreichend, denn bevor man Poisson anwenden kann, braucht man erst einmal die Wellenform. Eine andere Betrachtungsweise hilft weiter. Die Diskretheit des Signals - oder anders gesagt: die exakte Auslöschung der im Integral in (3) aufsummierten Beiträge für fast alle Emissionsrichtungen und Parameterwerte der einfallenden Wellen - kann letztendlich 
auf eine kontinuierliche Synmetrie dieser Wellen zurückgeführt werden, die zur diskreten Symmetrie der Struktur passt, aber über diese hinausgeht [5]. Die naive Idee, dass die durch das Integral in (3) mit der Elektronendichte ,verheiratete“ einfallende Welle nur dieselbe diskrete Translationssymmetrie haben müsste wie ihr Partner, kann ein Kontinuum von Auslöschungseffekten nicht erklären; eine kontinuierliche Symmetrie aber sehr wohl. Wie in [5] gezeigt, sind die elektromagnetischen ebenen Wellen (Gleichung (5)) gerade diejenigen Lösungen der zeitharmonischen Maxwell-Gleichungen, die kontinuierliche Translationssymmetrie haben, genauer: die simultane Eigenfunktionen der Operation der kontinuierlichen Translationsgruppe auf Vektorfeldern (siehe (4)) sind. Die Bedeutung dieser Symmetrie für die Beugungsmuster ist die folgende. Konstruktive Interferenz an den Beugungsmaxima beruht auf der Symmetrie der Wellen unter der diskreten Translationssymmetriegruppe der Struktur. Destruktive Interferenz abseits der Beugungsmaxima beruht auf der darüber hinausgehenden kontinuierlichen Symmetrie der Wellen.

Helixstrukturen wie in Bild I haben eine andere Symmetrie als Kristalle. Idealisiert man sie als unendlich lang, so ist ihre Elektronendichte invariant unter einer anderen diskreten Untergruppe der euklidischen Transformationen (4), nämlich einer Helixgruppe, gegeben durch die ganzzahligen Potenzen der erzeugenden diskreten Schraubentransformation. Die entsprechende kontinuierliche Helixgruppe besteht aus beliebigen Drehungen um und Translationen entlang der Schraubenachse. Dies legt nahe, von der einfallenden Welle - neben den zeitharmonischen Maxwell-Gleichungen (I), (2) - kontinuierliche Helixsymmetrie zu verlangen, d.h. die Eigenschaft, eine simultane Eigenfunktion der kontinuierlichen Helixgruppe zu sein. Hat man dieses Gleichungssystem für das Design von Röntgenwellen erst einmal aufgestellt, so gelangt man mithilfe klassischer Methoden der Darstellungstheorie und der Theorie linearer Differentialgleichungen zur Lösung. Das Resultat sind genau die twisted waves (6). Die Darstellungstheorie kommt hier ins Spiel, da sie die Eigenwerte der Operation (4) der Helixgruppe auf Vektorfeldern liefert.

Zur klassischen Röntgenkristallografie analoge Diskretheitseigenschaften des Röntgensignals von Helixstrukturen unter solchen twisted waves lassen sich mithilfe der Verallgemeinerung der Poisson-Formel auf abelsche Gruppen beweisen [5]. Diese Verallgemeinerung haben wir dem großen André Weil zu verdanken, der nicht durch Fragen der Strukturbiologie, sondern der Zahlentheorie motiviert war.

\section{Danksagung}

Ich bin Michael Joswig für die Anregung zu diesem Artikel und nützliche Hinweise zu einem ersten Entwurf zu Dank verpflichtet.

\section{Anmerkung}

I. Über Zuschriften von Leserinnen und Lesern, die das Gegenteil belegen, würden wir uns sehr freuen - wir haben monatelang vergeblich gesucht und bei Kollegen herumgefragt.

\section{Literatur}

[I] Protein Data Bank, http://www.rcsb.org, pdb entry 2tmv; K. Namba, R. Pattanayek, G. Stubbs, Visualization of proteinnucleic acid interactions in a virus. Refined structure of intact tobacco mosaic virus at $2.9 \AA$ resolution by $\mathrm{X}$-ray fiber diffraction. J. Mol. Biol. 208, 307-325, 1989

[2] Protein Data Bank in Europe, www.ebi.ac.uk/pdbe, entry EMD5259; R. H. Rochat, X. Liu, K. Murata, K. Nagayama, F.J. Rixon, W. Chiu, Seeing the portal in herpes simplex virus type I B capsids. J. Virology 85 (4), I87I-|874, 201 I

[3] Visualisierung: J.-Y. Sgro (www.virology.wisc.edu/virusworld/ viruslist.php?virus=hIb) via the UCSF Chimera package, developed by the Resource for Biocomputing, Visualization, and Informatics at the University of California, San Francisco (supported by NIGMS P4I-GMI033 I I); Pettersen EF, Goddard TD, Huang CC, Couch GS, Greenblatt DM, Meng EC, Ferrin TE, UCSF Chimera - a visualization system for exploratory research and analysis, J Comput Chem. 2004, Oct 25(I3): I605-I2.

[4] G.-M. Greuel, Kristalle und Mathematik, MDMV 22, 2।2-2।7, 20I4. (Eine schöne Einführung in Kristallstrukturen.)

[5] G. Friesecke, R.D. James, D. Jüstel, Twisted X-rays: incoming waveforms yielding discrete diffraction patterns for helical structures, SIAM J. Appl. Math. 76 (3), II9I-1218, 2016

[6] D. Jüstel, G. Friesecke, R.D. James, Bragg-von Laue diffraction generalized to twisted X-rays, Acta Cryst. A72, 190196, 2016. (Siehe auch news item der International Union of Crystallography, http://www.iucr.org/news/research-news/ twisted-x-rays-unravel-the-complexity-of-helical-structures)

[7] G. Friesecke, R. D. James, D. Jüstel. Manuskript in Vorbereitung.

[8] L. Allen, M. W. Beijersbergen, R. J. C. Spreeuw, J. P. Woerdman, Orbital angular momentum of light and the transformation of Laguerre-Gaussian laser modes, Phys. Rev. Lett. 45, 81858189,1992

[9] G. Friesecke, Lectures on Fourier Analysis, Vorlesungsskript (University of Warwick, 2007). Vom Autor erhältlich, gf@ma. tum.de

Prof. Dr. Gero Friesecke, Zentrum Mathematik, TU München, Boltzmannstraße 3, 85747 Garching. gf@ma.tum.de

Gero Friesecke ist seit 2004 Mathematikprofessor an der TUM, vorher war er als Professor an den Universitäten Oxford (1998-200I) und Warwick (200I-2004, in Teilzeit bis 2009) tätig. Er ist Träger des Oberwolfach-Preises sowie des Whitehead Prize der London Mathematical Society. Neben seiner Forschungstätigkeit engagiert er sich auf vielfältige Weise für die Interaktion zwischen Mathematik und Naturwissenschaften, z. B. als Mitglied des wissenschaftlichen Beirates des MPI für Mathematik in den Naturwissenschafen in Leipzig (www.mis.mpg.de) oder als Entwickler neuer Mathematik-Lehrprogramme für Studienanfänger in den Naturwissenschaften (aktueller Fernsehbeitrag: http://www.br.de/mediathek/video/sendungen/alpha-campus/ campus-mint-probe-eins-mathematik-100.html, Minute 7-14). 